\title{
Les politiques alimentaires face aux enjeux nutritionnels
}

\author{
Ambroise MARTIN ${ }^{1}$ \\ Louis-Georges SOLER ${ }^{2}$ \\ ${ }^{1}$ Faculté de médecine Grange Blanche, \\ JE2411 - Bureau 3/4300, \\ 8 , avenue Rockefeller, \\ 69373 Lyon cedex 08 \\ <ambroise.martin@sante.univ-lyon1.fr> \\ 2 INRA-LORIA, Unité ALISS, \\ 65 Boulevard de Brandebourg, \\ 94205 Ivry-sur-Seine cedex \\ <soler@ivry.inra.fr>
}

\begin{abstract}
Food policies can use of two intervention strategies. The first is changing consumer behaviour through information and education. The second is to act on the characteristics of the food supply, on the nutritonal composition of products in the same time on the prices. All require collaboration between governments and enterprises.
\end{abstract}

Key words: food policies, consumer behaviour, nutritional composition
Les effets des pratiques alimentaires sur la santé sont aujourd'hui bien établis. Afin de favoriser le développement d'actions préventives en matière d'alimentation, et de réduire ainsi les coûts liés à certains comportements de consommation, une première voie $d$ 'intervention de la part des pouvoirs publics consiste à tenter d'infléchir les choix alimentaires faits par les individus et les ménages. Dans de nombreux pays, c'est une des orientations importantes données aux politiques de santé et, en France, une grande partie des actions lancées dans le cadre du premier Plan national nutrition santé (PNNS1) se sont inscrites dans cette optique. Lancé en janvier 2001 pour une période de 5 ans, ce programme a surtout mis l'accent sur l'information et l'éducation du consommateur et des professionnels de santé. En témoignent les nombreux guides à destination du grand public ou de populations spécifiques (parents d'enfant de 0 à 18 ans, adolescents, personnes âgées, personnes précaires, femmes enceintes) et leurs livrets d'accompagnement destinés aux professionnels de santé (www. mangerbouger.fr). Ces livrets visent à faire prendre conscience de l'importance des choix alimentaires pour la santé et proposent de nombreuses pistes pour effectuer des choix judicieux pour l'équilibre alimentaire.

L'évaluation des actions conduites dans le PNNS1 va bénéficier des données en cours d'exploitation des enquêtes nationales représentatives INCA2/ENNS réalisées par l'Afssa et I'InVS. Mais anticipant, comme le montrent diverses études sur le plan international, un impact modeste des actions d'information des consommateurs, des actions visant à faire évoluer l'offre alimentaire ont été proposées dans le cadre du second Plan national nutrition santé
(PNNS2), renouvelé pour la période 20062010.

Dans le cadre du PNNS2, diverses pistes ont été envisagées pour agir sur l'offre alimentaire de façon à contribuer à l'amélioration de l'état nutritionnel des populations, en particulier celles aux revenus les plus modestes qui sont le plus sensibles à ces enjeux de santé. Tout en poursuivant les actions d'information et d'éducation déjà engagées, le PNNS2 porte ses efforts sur l'évolution de l'offre alimentaire, considérant qu'il est important que les choix judicieux puissent être facilités par la disponibilité et l'accessibilité des produits plus conformes aux recommandations nutritionnelles. $\mathrm{Ce}$ sont ces orientations données au PNNS2 qui conduisent à soulever la question des relations entre les politiques nutritionnelles, agricoles et alimentaires.

D'une certaine manière, I'offre alimentaire est déjà concernée par les évolutions souhaitées par les politiques de santé publique en matière de comportement de consommations. II est clair que si l'ensemble des consommateurs adoptait des pratiques alimentaires conformes aux recommandations nutritionnelles, le déplacement de la demande conduirait nécessairement à des réajustements au niveau de I'offre alimentaire. Une étude conduite aux USA a ainsi montré des impacts significatifs, tant sur les volumes et les prix des divers produits agricoles que sur la localisation géographique des productions. Mais la question qui est désormais soulevée en diverses instances, et particulièrement en France par le PNNS2, est celle de la contribution possible de l'évolution de l'offre alimentaire à l'adoption de pratiques alimentaires plus conformes aux enjeux nutritionnels.
Quels arguments peuvent être avancés pour justifier une adaptation de l'offre alimentaire de ce point de vue? Certains économistes soutiennent en effet que dès lors que les consommateurs sont informés, à la fois des caractéristiques des aliments qui leur sont proposés et des risques/bénéfices associés à leur consommation, c'est à eux de faire les bons arbitrages et non pas aux entreprises de modifier les prix ou les caractéristiques des aliments pour des raisons de santé publique. Plusieurs réponses peuvent être données à cette position, mais la principale tient aux disparités économiques et de revenus. Même si tous les consommateurs étaient parfaitement informés sur le plan nutritionnel, pourraient-ils tous pour autant assurer une alimentation équilibrée? La réponse à cette question est plutôt négative :

- d'une part, du fait de la structure relative des prix des diverses catégories d'aliments ;

- d'autre part, du fait que pour une catégorie d'aliments donnée, des niveaux plus élevés de qualité nutritionnelle peuvent être associés à des niveaux de prix plus élevés.

Si l'on admet donc, pour ces diverses raisons, que l'évolution de l'offre alimentaire constitue une piste d'action, parmi d'autres, pour favoriser des pratiques alimentaires plus conformes aux enjeux nutritionnels, la question se pose alors des modalités d'intervention dans ce domaine. On discute dans cet article les diverses pistes d'intervention dans ce sens.

\section{Agir sur la structure relative des prix ?}

Des actions au long cours pour une évolution de la structure des prix relèvent d'une politique économique et alimentaire qui nécessite sans 
doute encore beaucoup de réflexions et de travaux. On peut à ce stade soulever les points suivants en distinguant la question des prix des produits alimentaires transformés et celle des prix des produits agricoles.

\section{L'action sur les prix des aliments transformés}

Les effets des prix sur les consommations alimentaires ont été examinés dans de nombreux travaux économiques. C'est sur cette base que la question de la taxation "nutritionnelle » a été soulevée : elle viserait à modifier, en les rendant plus chers, la consommation de groupes de produits ou de nutriments particuliers supposés être responsables des déséquilibres alimentaires. Elle peut être fondée sur la teneur en un seul nutriment (sucre, graisse, etc.) ou, comme cela a été plus récemment proposé, sur un profil nutritionnel plus complexe [1].

L'intérêt de cette action sur les prix est très controversé. Pour certains auteurs, les effets $d^{\prime}$ 'une telle taxe pourraient être significatifs, mais d'autres études soulignent les difficultés liées à a mise en œuvre d'une telle démarche : - Elle pourrait induire des effets différenciés selon les catégories sociales, les augmentations de prix, du fait des substitutions qu'elles induisent, ayant des effets négatifs sur les catégories disposant des plus faibles revenus.

- Les réels effets sont difficilement prévisibles: d'un côté, si la taxe n'est pas assez exigeante (sur l'assiette et le taux), on constate qu'elle a peu d'effets sur les volumes et les substitutions ; d'un autre côté, si elle est très exigeante, elle peut induire des gammes de substitution entre produits qui ne sont pas nécessairement celles qui sont recherchées.

- Enfin, elle peut conduire certains opérateurs à dégrader la qualité des produits pour réduire les coûts de production et compenser l'effet de hausse des prix lié à la taxe.

\section{Les prix de produits agricoles}

Même si d'autres interventions publiques affectent les prix des produits alimentaires (politiques publiques en matière de qualité et de sécurité des produits, politique de la concurrence et des pratiques commerciales, etc.), la PAC est parfois incriminée pour ses effets négatifs sur la santé nutritionnelle des populations européennes.

On peut déjà remarquer qu'il n'y a pas de relation directe entre la PAC, les soutiens publics à l'agriculture et les problèmes nutritionnels observés aujourd'hui. Des pays comme le Japon qui a subventionné son agriculture connaît moins de problèmes d'obésité que des pays qui ont des marchés plus ouverts. Au sein même de l'UE, qui a mis en place une politique agricole commune, les situations sont variées sur le plan du développement de l'obésité.

Par ailleurs, les politiques agricoles s'inscrivent dans une tendance générale plutôt orientée vers la diminution des soutiens publics et la baisse des prix. La dimension nutritionnelle devrait-elle être introduite dans ce cadre, par exemple, pour freiner ou accélérer cette évolution selon les produits agricoles?

Cette question doit être examinée de façon différente selon que les produits agricoles sont utilisés à des fins de transformation industrielle ou consommés en l'état par les consommateurs.

Concernant les produits agricoles destinés à la transformation, la nécessité d'intervenir sur les prix de la matière première pour des raisons nutritionnelles n'est pas évidente. Les rapports de prix entre matières premières affectent les arbitrages au niveau industriel sur le plan de la formulation des produits. Néanmoins, la part de la matière première sur le prix du produit final est généralement faible. D'autre part, les contraintes technologiques et sensorielles limitent les marges de manœuvre au niveau industriel. Des recherches en cours visent à étudier les effets possibles de la baisse attendue du prix du sucre du fait de la réforme de la politique sucrière au niveau européen sur les choix de formulation (Projet ANR Polnutrition, P. Bomtems - Inra).

La question est différente dans le cas des produits agricoles consommés en l'état, en particulier les fruits et légumes ( $F \& L$ ). Les études convergent pour indiquer qu'il s'agit d'une catégorie de produits dont l'importance d'achat est conditionnée par des critères économiques, même si d'autres critères interviennent, comme la capacité à savoir les préparer, notamment pour les légumes. Les études portant sur la relation entre la qualité nutritionnelle des aliments et leur prix démontrent clairement l'existence d'une relation inverse entre les deux paramètres [2]. Ces études montrent que le coût de la calorie est 5 fois plus élevé pour cette catégorie que pour les autres; même si elles montrent aussi qu'ils comportent en moyenne 5 fois plus de nutriments intéressants, ce qui les met au même prix que les autres en termes d'intérêt nutritionnel, cet argument pèse peu quand les contraintes de revenus sont présentes.

Plusieurs éléments déterminent le niveau des prix des F\&L (cf. le rapport INRA ESCO Fruits et Légumes, 2007). Si I'on s'en tient ici à la politique agricole européenne, il faut noter de grandes différences entre le secteur des F\&L et d'autres secteurs comme ceux des céréales ou du lait. Dans ces derniers, la réforme de la PAC a fondamentalement consisté à favoriser des baisses de prix en accompagnant les exploita- tions par des aides directes de façon à compenser, dans une certaine mesure les baisses de revenus. Le secteur des F\&L n'a pas bénéficié de soutiens directs importants. Jusqu'au milieu des années 90, des mécanismes étaient en place au niveau européen pour éviter de trop fortes baisses des cours susceptibles de mettre en difficulté les producteurs. II s'agissait principalement de démarches de «retraits» qui consistaient, lors de périodes d'excédents, à retirer du marché des quantités importantes de fruits afin de soutenir les prix payés aux producteurs. Des barrières douanières (moins importantes que pour d'autres productions agricoles) et des limitations des importations dans les périodes de production en Europe protégeaient également les exploitations.

Les évolutions des dernières années ont consisté à réduire, pour in fine les faire disparaître, ces mécanismes de retrait qui maintenaient de mauvais ajustements entre l'offre et la demande. Mais la disparition de ces mécanismes de protection a mis en face à face les producteurs (très atomisés) et les distributeurs (très concentrés). Les conflits récurrents entre la grande distribution et les producteurs ont conduit les pouvoirs publics français à instaurer de nouveaux mécanismes pour la protection des producteurs.

Dans ce contexte, il apparaît que, malgré un réel dynamisme du secteur qui s'est traduit par des gains de productivité significatifs, la baisse des prix en aval des producteurs a été moins forte pour les $F \& L$ que dans d'autres secteurs. Ainsi, les fruits et légumes, produits peu soutenus de manière générale, n'ont pas connu le processus de réforme à l'œuvre depuis 1992, visant la baisse du soutien par les prix et la compensation des pertes induites de revenu par des aides directes.

\section{Agir sur les caractéristiques des aliments?}

\section{Les produits agricoles peu ou pas transformés}

Une première voie d'action pour l'amélioration de la valeur nutritionnelle des aliments réside dans l'innovation et les démarches de recherche-développement. Elles peuvent s'appliquer aux produits transformés mais aussi aux produits agricoles bruts. Le cas de la viande est illustratif.

II s'agit des travaux qui étudient l'impact des techniques agricoles de culture ou d'élevage sur la qualité nutritionnelle des produits agricoles remis au consommateur. Jusqu'à maintenant, il était exceptionnel que des critères nutritionnels soient pris en compte dans les évolutions de ces pratiques au côté des critères essentiels que sont les valeurs agronomiques et 
technologiques et leur impact sur le prix, qui devait toujours tendre vers le bas, sous la pression de la concurrence mondiale. Ainsi, des réflexions ont été conduites à l'Afssa sur le lien les pratiques zootechniques et la qualité des produits alimentaires; commencées avec la problématique, plutôt sur un versant sécuritaire, des vitamines liposolubles dans l'élevage des jeunes animaux [3], elles se sont poursuivies sur le sujet de la nature des acides gras (un fort déséquilibre existant en France entre les séries oméga 6 et 3, [4]) et l'iode (la France étant toujours un pays considéré par l'OMS comme à risque de carence modérée en iode [5]). Elles pourraient être étendues à d'autres problématiques concernant les minéraux et oligoéléments par exemple. Ces travaux reconnaissent que, si le constat scientifique est relativement aisé à faire, la mise en place d'évolutions risque d'être très difficile en I'absence de réelle politique globale. Des filières particulières se mettent parfois en place sur de tels critères, mais elles restent encore quantitativement marginales. II en est de même sur le versant végétal: des réflexions ont été conduites à l'Académie d'agriculture sur l'éventuelle prise en compte de critères nutritionnels dans la sélection variétale des végétaux [6]: des études montrent que sur des espèces importantes comme le blé, des différences de l'ordre de trois fois peuvent être constatées entre les variétés pour les fibres ou les minéraux, qui se retrouvent dans les produits finis comme la farine et le pain [7].

\section{Pour les produits alimentaires transformés}

\section{Allégations, étiquetage et profils nutritionnels}

L'étiquetage nutritionnel vise à informer les consommateurs sur les caractéristiques des produits qui leur sont proposés. Les allégations permettent aux entreprises de mettre en avant un allégement ou un enrichissement en un nutriment, censé améliorer la qualité nutritionnelle d'un produit. La nouvelle réglementation européenne [8] vise à garantir aux consommateurs, d'une part, que le produit qui porte une allégation est sensiblement différent du produit standard, d'autre part, que l'amélioration sur la teneur en un nutriment ne cache pas des caractéristiques défavorables sur les autres nutriments.

La mise en place des profils nutritionnels, définis dans le règlement européen 1924/2006 sur la régulation des allégations nutritionnelles et de santé, s'inscrit dans cette perspective. Ce règlement prévoit que seuls les aliments respectant un certain profil (et notamment pas trop riches en lipides, sucres ou sel) pourront porter des allégations. Les allégations représentent un outil promotionnel important pour l'industrie, confirmé par l'enquête DGAL/CLCV [9], montrant qu'elles constituent une information importante dans la décision d'achat, bien avant la liste des ingrédients ou l'étiquetage nutritionnel. De ce fait, de plus en plus de produits portent des allégations (les trois quarts des nouveaux produits mis sur le marché au niveau mondial). Le législateur européen a considéré qu'il était nécessaire que la présence d'une allégation valorisante ne puisse pas masquer la composition éventuellement globalement défavorable d'un produit alimentaire. II a estimé aussi que ces profils pouvaient être un encouragement à l'innovation et à la reformulation des produits.

Dans une logique d'entreprise industrielle ou de distribution cependant, les produits avec allégations s'inscrivent dans des stratégies de différenciation et de segmentation du marché. La prime à l'innovation conduit à positionner ces produits sur des niveaux de prix plus élevés, à destination de consommateurs ayant de plus fortes dispositions à payer. Les reformulations vers des produits plus sains sur le plan nutritionnel pourraient alors ne toucher que des produits de niche, à forte valeur ajoutée, même si on ne peut exclure un effet général d'entraînement pour l'ensemble des produits.

On peut noter que la circulaire sur la restauration collective publique et notamment scolaire comporte aussi des profils nutritionnels, qui conditionnent les fréquences de proposition des différents composants dans les menus. Cependant, cette recommandation n'a qu'un poids modeste et est très loin d'être appliquée de façon satisfaisante. Parmi les écueils à son application, les contraintes économiques sont là encore présentes.

\section{Normalisation et réglementation des caractéristiques des produits}

Pour intervenir sur les caractéristiques des produits de l'offre alimentaire de base, une autre voie réside dans la réglementation et l'imposition de normes. De telles démarches ont été envisagées en diverses instances, pour le sucre et le sel par exemple. Elles soulèvent de nombreux débats et conflits. II n'existe pas, en effet, à notre connaissance de travaux économiques sur les effets des réglementations nutritionnelles sur les performances des entreprises et des filières. Or les effets induits par ces démarches de normalisation peuvent selon les cas s'avérer positifs, négatifs ou nuls.

Les interventions des pouvoirs publics ont nécessairement des conséquences économiques sur les acteurs des filières de production transformation - commercialisation, au tout premier plan desquels les entreprises agricoles et industrielles fabricant les produits « incriminés ». Elles peuvent en effet induire, selon les cas une augmentation des coûts de fabrica- tion, une modification du jeu concurrentiel, une modification des stratégies de segmentation du marché.

Ces conséquences doivent être identifiées. Elles dépendront :

- des surcoûts induits par le resserrement des normes ;

- de la structure de la concurrence à chaque maillon des chaînes alimentaires (hétérogénéité et concentration des entreprises, degré de différenciation des produits);

- des types de relations et les rapports de force entre les opérateurs d'amont et d'aval.

Tous ces éléments jouent sur les parts de marché, le partage de la valeur, la transmission des prix aux consommateurs et le niveau de qualité des produits mis en marché. Ils doivent être évalués pour orienter correctement les interventions publiques. Si on augmente la contrainte réglementaire, les surcoûts induits peuvent, dans certains cas, être transmis sur le marché final. Les hausses des prix à la consommation peuvent alors induire un report des consommateurs vers des produits présentant des caractéristiques nutritionnelles moins bonnes.

\section{Chartes d'engagement et observatoire de la qualité de I'alimentation}

Si on ne peut nier l'importance d'une adaptation de l'offre alimentaire face aux enjeux nutritionnels, il apparaît qu'il n'existe pas de solutions simples permettant $d^{\prime}$ influencer dans ce sens, et de façon directe, les mécanismes de marché. Dans un tel contexte, une voie à privilégier consiste, pour les pouvoirs publics, à agir de façon à conduire les acteurs privés à engager des actions «volontaires» allant dans le sens d'une amélioration de la qualité nutritionnelle de I'alimentation.

C'est dans cette voie que s'est engagé le PNNS2 en proposant la mise en place de chartes d'engagements contractuels entre l'État et les entreprises ou les interprofessions. Le référentiel précise que ces engagements doivent concerner une part majoritaire de la production de l'entreprise et des critères de prix seront pris en considération. Ces engagements permettront aux professionnels de valoriser auprès du consommateur le sérieux de leur démarche dans le domaine de la nutrition. La possibilité de contrôle par tierce partie du respect des engagements est un élément fondamental. Pour le moment, aucune charte n'a encore été signée et il faudra évidemment évaluer leur impact en ce qui concerne l'accessibilité d'aliments plus intéressants pour la santé. Ces chartes représentent une expérimentation intéressante qui pourrait favoriser des évolutions spontanément difficiles, comme le montre la très lente évolution de la teneur en sel du pain. 
En complément des chartes d'engagement, la création d'un observatoire de la qualité a été proposée de façon à suivre les actions engagées par les entreprises dans la perspective de l'amélioration de la qualité nutritionnelle de leur offre alimentaire. II s'agit ainsi d'objectiver les évolutions des caractéristiques de l'alimentation et de dresser périodiquement un état des lieux en matière de qualité nutritionnelle des aliments et de mettre en évidence les efforts accomplis par les entreprises dans ce sens.

Si I'on admet que, dans ce domaine, il sera longtemps difficile d'établir des principes clairs de responsabilité sur le plan juridique, la nécessité d'associer intervention publique et actions volontaires des entreprises apparaît clairement. Une intervention de la part des seuls pouvoirs publics exigerait des moyens importants, serait confrontée à de grandes difficultés dans sa mise en œuvre opérationnelle et générerait forcément de multiples stratégies de contournement par les entreprises. D'un autre côté, les actions volontaires conduites par les entreprises présentent un intérêt, mais sans incitations mises en place par les pouvoirs publics elles pourraient ne concerner que des segments de produits différenciés et non pas les segments de produits destinés aux catégories les plus confrontées aux risques nutritionnels.

L'appel à des démarches volontaires ne peut déboucher sur des avancées significatives que $s^{\prime}$ il est associé à des dispositifs de pilotage et de contrôle, portés par les pouvoirs publics ou par des instances indépendantes, qui orientent les stratégies industrielles dans le sens des objectifs de santé publique.

\section{RÉFÉRENCES}

1. MYTTON O, GRAY A, RAYNER M, RUTTER H Could targeted food taxes improve health? J Epidemiol Community Health 2007 ; 61 : 68994

2. MAILLOT M, DARMON N, DARMON M, LAFAY L, DREWNOWSKI A. Nutrient-dense food groups have high energy costs: an econometric approach to nutrient profiling. I Nutr 2007 ; $137: 1815-20$.

3. AFSSA. Évaluation des besoins nutritionnels des animaux en vitamines $A$, $D$ et $E$ ainsi que des risques pour la santé animale et la santé du consommateur, liés à des apports élevés chez les animaux producteurs d'aliments. 2003.
4. AFSSA. Acides gras de la famille oméga 3 et système cardiovasculaire : intérêt nutritionnel et allégations. Rapport, juillet 2003.

5. AFSSA. Évaluation de l'impact nutritionnel de l'introduction de composés iodés dans les produits agroalimentaires. Rapport de mars 2005.

6. MARTIN A. Pour une prise en compte de critères nutritionnels dans la sélection variétale des végétaux. Comptes rendus des séances de I'Académie d'agriculture, séance du 13 février 2002. www.academie-agriculture.fr/seances/ 2002.

7. OURY FX, LEENHARDT F, REMESY C, ET AL. Genetic variability and stability of grain magnesium, zinc and iron concentrations in bread wheat. Eur J Agron $2006 ; 25$ : 177-85.

8. Règlement $1924 / 2006$ du 20 décembre 2006 concernant les allégations nutritionnelles et de santé portant sur les denrées alimentaires. Rectificatif publié au JOCE du 18 janvier 2007 L12/3-18.

9. DGAL/CLCV. Convention A02/22 relative à l'étude de la compréhension par les consommateurs de certaines mentions figurant dans l'étiquetage des denrées alimentaires préemballées et à leur perception de certaines allégations nutritionnelles, fonctionnelles et de santé. 2004. 